\title{
ON THE SOLUTION OF THE GENERAL SINGULAR MODEL OF 2-D SYSTEMS
}

\author{
Nicholas P. Karampetakis* \\ * Department of Mathematics, Aristotle University of \\ Thessaloniki, Thessaloniki 54006, Greece, \\ email:karampet@math.auth.gr
}

\begin{abstract}
The main objective of this work is to provide a closed formula for the forward, backward and symmetric solution of the 2-D general singular model (GSM) given in (Kurek, 1985). Of fundamental importance in our approach is the relative forward and backward fundamental matrix. Copyright ${ }^{\complement} 2005$ IFAC.
\end{abstract}

Keywords: 2-D general singular models, implicit 2-D systems, fundamental matrix, forward solution, backward solution, symmetric solution

\section{INTRODUCTION}

Consider the 2-D linear discrete time systems proposed in (Kaczorek, 1988) as a generalization of the 2-D state-space model given in (Kurek, 1985)

$$
\begin{gathered}
E x(i+1, j+1)=A_{0} x(i, j)+ \\
A_{1} x(i+1, j)+A_{2} x(i, j+1)+B_{0} u(i, j)+ \\
+B_{1} u(i+1, j)+B_{2} u(i, j+1)
\end{gathered}
$$

where $i, j$ are integer-value vertical and horizontal coordinates, respectively, $x(i, j) \in \mathbb{R}^{n}$ is the local state vector at $(i, j), u(i, j) \in \mathbb{R}^{m}$ is the input vector, $A_{k} \in \mathbb{R}^{n \times n}, B_{k} \in \mathbb{R}^{n \times m}, k=0,1,2$ and matrices $E, A_{0} \in \mathbb{R}^{n \times n}$ exists and are not necessarily nonsingular. This model includes similar generalization of other 2-D state space models such as the Fornasini and Marchesini (Fornasini and Marchesini, 1970) and the Roesser 2-D model (Roesser, 1975). If $E \neq I$ we call these models implicit 2-D systems. We shall call (1) the general singular model (GSM) or otherwise the implicit Fornasini-Marchesini (FM) model. If $E$ is non-square or $\operatorname{det}(E)=0$ we call these models singular 2-D systems. One particular case of (1) is the implicit Roesser model proposed in (Kaczorek, 1987) and (Lewis, 1987) as a generalization of the Roesser 2-D model given in
(Roesser, 1975). It is shown in (Kaczorek, 1989) that the implicit Roesser and the implicit FM model are equivalent. Due to the equivalence of the above models we consider in the rest of the paper only the GSM model. An example of the GSM model is providing by discretizing the heat equation using the method of central differences (Karamancioglu, 1991), while an example of the implicit Roesser model is given by the 2-D realization of a nonrecursible mask in digital image processing (Lewis and Mertzios, 1991). Implicit FM models are also arising from the discretization of continuous-time systems that are described by partial differential equations i.e. the standard discretization of the elliptic equation that results in a five-point discrete mask or the discretization of the diffusion equation that results in a four-point discrete mask (Karamancioglu, 1991).

According to (Lewis, 1992) there are various ways to specify the boundary conditions (BCs) and the region of interest for the FM and Roesser models. First suppose that the 2-D implicit system has BCs specified along the $i-$ and $j-$ axes. For the GSM model this means we know :

$$
\begin{gathered}
x(i, 0)=x_{i 0}, i=0,1, \ldots, N \\
x(0, j)=x_{0 j}, j=0,1, \ldots, M
\end{gathered}
$$


where $x_{i 0}$ and $x_{0 j}$ are known vectors. Then, if the region of interest is the rectangle $[0, N] \times[0, M]$ in the $(i, j)$-plane, we are concerned with finding what could be called a "forward solution ". If the BCs are specified along the upper and right-hand sides of the rectangle :

$$
\begin{aligned}
& x(i, M)=x_{i M}, i=0,1, \ldots, N \\
& x(N, j)=x_{N j}, j=0,1, \ldots, M
\end{aligned}
$$

then the solution on $[0, N] \times[0, M]$ could be called "backward solution ". A general case which includes both of these situations is where the BCs are of the split or two-point form :

$$
\begin{array}{ll}
C_{i, 0}^{u} x(i, 0)+C_{i, M}^{u} x(i, M)=c_{i}^{u}, & 0 \leq i \leq N \\
C_{0, j}^{h} x(0, j)+C_{N, j}^{h} x(N, j)=c_{i}^{h}, & 0 \leq j \leq M
\end{array}
$$

with $\left[C_{i, 0}^{u} C_{i, M}^{u}\right]$ and $\left[C_{0, j}^{h} C_{N, j}^{h}\right]$ prescribed matrices of full row rank and $c_{i}^{u}, c_{i}^{h}$ given vectors. If the BCs are of the split form given above or otherwise involve the semistate along all boundaries of the rectangular region $[0, N] \times[0, M]$ then the solution on $[0, N] \times[0, M]$ could be called "symmetric solution ".

A complete analysis of solutions and properties in the forward, backward and symmetric case for the 1-D singular systems $E x(i+1)=A x(i)+$ $B u(i)$ was given in (Lewis and Mertzios, 1990) in terms of the matrices $E, A, B$ and the forward and backward fundamental matrix of $(z E-A)^{-1}$. (Lewis and Mertzios, 1991) and (Kaczorek, 1990) have proposed, a forward solution to the 2-D implicit Roesser model and GSM respectively, in terms of the forward fundamental matrix of the system. Following similar methods to those of (Lewis and Mertzios, 1990), we produce a closed formula for the backward and symmetric solution of the GSM (1) in terms of the forward fundamental matrix $T_{p, q}$ and backward fundamental matrix $\tilde{T}_{p, q}$ of $\left(z_{1} z_{2} E-A_{0}-A_{1} z_{1}-A_{2} z_{2}\right)^{-1}$. A generalized Leverrier technique for computing the forward fundamental matrix sequence is available (Mertzios and Lewis, 1988), (Karampetakis et al., 1994), so that we may assume that this matrix sequence is given. We shall show in Section 2 that the backward fundamental matrix sequence of $z_{1} z_{2} E-A_{0}-A_{1} z_{1}-A_{2} z_{2}$ is the forward fundamental matrix sequence of the dual polynomial matrix $E-A_{0} z_{1} z_{2}-A_{1} z_{2}-A_{2} z_{1}$, and thus we may assume that the backward fundamental matrix sequence is also given.

\section{PRELIMINARY RESULTS}

Assuming that the polynomial matrix

$$
G\left(z_{1}, z_{2}\right)=z_{1} z_{2} E-A_{0}-A_{1} z_{1}-A_{2} z_{2}
$$

with $E \neq 0$, is invertible, the Laurent expansion at infinity of $G\left(z_{1}, z_{2}\right)^{-1}$ exists, is unique (Karampetakis et al., 1994), and is given by :

$$
G\left(z_{1}, z_{2}\right)^{-1}=\sum_{p=-n_{1}}^{\infty} \sum_{q=-n_{2}}^{\infty} T_{p, q} z_{1}^{-p} z_{2}^{-q}
$$

$\left(n_{1} \leq n, n_{2} \leq n\right)$ and $\left|z_{1}\right|>\sigma_{1}>0,\left|z_{2}\right|>\sigma_{2}>0$ where the matrix sequence $\left\{T_{p, q}\right\}$ is known as the forward fundamental matrix. Note that a necessary and sufficient condition for the uniqueness of the fundamental matrix sequence is that condition $\operatorname{deg}_{z}|G(z, z)|=\operatorname{deg}_{z_{1}}\left|G\left(z_{1}, z_{2}\right)\right|+$ $\operatorname{deg}_{z_{2}}\left|G\left(z_{1}, z_{2}\right)\right|$ is satisfied (Karampetakis et al., 1994). Let also the Laurent expansion about zero of $G\left(z_{1}, z_{2}\right)^{-1}$ exists, is unique and is given by

$$
\begin{gathered}
G\left(z_{1}, z_{2}\right)^{-1}=\sum_{p=\ell_{1}}^{-\infty} \sum_{q=\ell_{2}}^{-\infty} V_{p, q} z_{1}^{-p} z_{2}^{-q} \\
\left|z_{1}\right|<\sigma_{1},\left|z_{2}\right|<\sigma_{2}
\end{gathered}
$$

where the matrix sequence $\left\{V_{p, q}\right\}$ is known as the backward fundamental matrix. The Laurent expansion about zero of $G\left(z_{1}, z_{2}\right)^{-1}$ given in (7) is related with the Laurent expansion at infinity given in (6) of the inverse of the dual matrix

$$
\begin{aligned}
\tilde{G}\left(z_{1}, z_{2}\right) & =E-A_{0} z_{1} z_{2}-A_{1} z_{2}-A_{2} z_{1} \equiv \\
& \equiv z_{1} z_{2} G\left(\frac{1}{z_{1}}, \frac{1}{z_{2}}\right)
\end{aligned}
$$

as we can see in the following Lemma.

Lemma 1. Let the Laurent expansion at infinity of $\tilde{G}\left(z_{1}, z_{2}\right)^{-1}$ be

$$
\tilde{G}\left(z_{1}, z_{2}\right)^{-1}=\sum_{p=-f_{1}}^{\infty} \sum_{q=-f_{2}}^{\infty} \tilde{T}_{p, q} z_{1}^{-p} z_{2}^{-q}
$$

and (7) be the Laurent expansion at zero of $G\left(z_{1}, z_{2}\right)^{-1}$. Then

$$
\begin{array}{r}
\quad f_{i}+1=\ell_{i} \text { and } V_{-i,-j}=\tilde{T}_{i+1, j+1} \\
i=\ell_{1}, \ell_{1}-1, \ldots \text { and } j=\ell_{2}, \ell_{2}-1, \ldots
\end{array}
$$

Proof. We have that

$$
\begin{gathered}
G\left(z_{1}, z_{2}\right)^{-1}=z_{1}^{-1} z_{2}^{-1} \tilde{G}\left(\frac{1}{z_{1}}, \frac{1}{z_{2}}\right)^{-1}= \\
=\sum_{p=-f_{1}}^{\infty} \sum_{q=-f_{2}}^{\infty} \tilde{T}_{p, q} z_{1}^{p-1} z_{2}^{q-1} \equiv \sum_{p=\ell_{1}}^{\infty} \sum_{q=\ell_{2}}^{\infty} V_{p, q} z_{1}^{-p} z_{2}^{-q}
\end{gathered}
$$

Equating the coefficients of the powers of $z_{i}, i=$ 1,2 we obtain the proof of Lemma.

We conclude from the above Lemma that the Laurent expansion at zero of $G\left(z_{1}, z_{2}\right)^{-1}$ exists and is unique iff the Laurent expansion at infinity of $\tilde{G}\left(z_{1}, z_{2}\right)^{-1}$ exists and is unique or otherwise when $\tilde{d}_{d}^{u}=\tilde{d}_{1}^{u}+\tilde{d}_{2}^{u}$, where

$$
\begin{aligned}
& \tilde{d}_{i}^{u}=\operatorname{deg}_{z_{i}}\left|E-A_{0} z_{1} z_{2}-A_{1} z_{2}-A_{2} z_{1}\right|, i=1,2 \\
& \tilde{d}_{d}^{u}=\operatorname{deg}_{z}\left|E-A_{0} z^{2}-\left(A_{1}+A_{2}\right) z\right|
\end{aligned}
$$


We can easily check that this condition is equivalent to the condition $d_{d}^{d}=d_{1}^{d}+d_{2}^{d}$, where $d_{i}^{d}$ is the less power of $z_{i}$ in $\operatorname{det}\left(G\left(z_{1}, z_{2}\right)\right)$ and $d_{d}^{d}$ is the less power of $z$ in $\operatorname{det}(G(z, z))$ i.e. $d\left(z_{1}, z_{2}\right)=$ $\operatorname{det}\left(G\left(z_{1}, z_{2}\right)\right)=\sum_{i=d_{1}^{d}}^{d_{i}^{u}} \sum_{j=d_{2}^{d}}^{d_{2}^{u}} d_{i, j} z_{1}^{i} z_{2}^{j}$. A direct result of Lemma 1 is that the Leverrier algorithm presented in (Karampetakis et al., 1994) may be used for the computation of both the forward and backward fundamental matrix sequence. An interesting result that connects the solutions of (1) and the ones of the dual 2-D GSM

$$
\begin{gathered}
E \tilde{x}(i, j)=A_{0} \tilde{x}(i+1, j+1)+\quad(12) \\
+A_{1} \tilde{x}(i, j+1)+A_{2} \tilde{x}(i+1, j)+B_{0} \tilde{u}(i+1, j+1)+ \\
+B_{1} \tilde{u}(i, j+1)+B_{2} \tilde{u}(i+1, j)
\end{gathered}
$$

in the closed interval $[0, N] \times[0, M]$ is given by the following Lemma.

Lemma 2. (a) If $\tilde{x}(i, j)$ is a solution of (12) for the non-zero input $\tilde{u}(i, j)$, then the sequence $x(i, j)=\tilde{x}(N-i, M-j)$ is a solution of the dual equation (1) for the nonzero input $u(i, j)=$ $\tilde{u}(N-i, M-j)$. (b) If $x(i, j)$ is a solution of (1) for the non-zero input $u(i, j)$, then the sequence $\tilde{x}(i, j)=x(N-i, M-j)$ is a solution of the dual equation (12) for the nonzero input $\tilde{u}(i, j)=$ $u(N-i, M-j)$.

Proof. (a) Let $\tilde{x}(i, j)$ be a solution of (12) for the non-zero input $\tilde{u}(i, j)$. This implies that (12) is satisfied. Now consider equation (1). If we set $x(i, j)=\tilde{x}(N-i, M-j), u(i, j)=$ $\tilde{u}(N-i, M-j)$ we have

$$
\begin{gathered}
E x(i+1, j+1)=E \tilde{x}(N-(i+1), M-(j+1))= \\
\stackrel{(12)}{=} A_{0} \tilde{x}(N-(i+1)+1, M-(j+1)+1)+ \\
\quad+A_{1} \tilde{x}(N-(i+1), M-(j+1)+1)+ \\
\quad+A_{2} \tilde{x}(N-(i+1)+1, M-(j+1))+ \\
+B_{0} \tilde{u}(N-(i+1)+1, M-(j+1)+1)+ \\
+B_{1} \tilde{u}(N-(i+1), M-(j+1)+1)+ \\
\quad+B_{2} \tilde{u}(N-(i+1)+1, M-(j+1))= \\
(x(i, j)=\tilde{x}(\stackrel{N}{=}-i, M-j)) A_{0} x(i, j)+A_{1} x(i+1, j)+ \\
u(i, j)=\tilde{\bar{u}}(N-i, M-j) \\
\quad+A_{2} x(i, j+1)+B_{0} u(i, j)+ \\
\quad+B_{1} u(i+1, j)+B_{2} u(i, j+1)
\end{gathered}
$$

(b) In the same way we can prove the second part of the Theorem.

A direct result of Lemma 2 is that the backward solution of the GSM (1) comes directly from the forward solution of the dual GSM (12).

\section{SOLUTIONS OF GSMS}

In the next three subsections we give the forward, backward and symmetric solution of the GSM (1) in terms of the matrix coefficients $A_{i}, B_{i}, i=$ $0,1,2$ and the forward/backward fundamental matrix sequence $\left\{T_{p, q}\right\} /\left\{V_{p, q}\right\}$ of $G\left(z_{1}, z_{2}\right)^{-1}$.

\subsection{The forward solution of general singular models}

Consider the GSM (1) and the Laurent matrix expansion at infinity of $G\left(z_{1}, z_{2}\right)^{-1}$ given in (6). Then the unique forward solution to (1) with admissible (2) is given according to (Kaczorek, 1990) by :

$$
\begin{gathered}
x(i, j)=\sum_{p=0}^{i+n_{1}} \sum_{q=0}^{j+n_{2}} T_{i-p, j-q} B_{0} u(p, q)+ \\
+\sum_{p=0}^{i+n_{1}+1} \sum_{q=0}^{j+n_{2}} T_{i-p+1, j-q} B_{1} u(p, q)+ \\
+\sum_{p=0}^{i+n_{1}} \sum_{q=0}^{j+n_{2}+1} T_{i-p, j-q+1} B_{2} u(p, q)- \\
-\sum_{p=0}^{i+n_{1}} T_{i-p, j+1}\left[A_{2} B_{2}\right]\left[\begin{array}{l}
x(p, 0) \\
u(p, 0)
\end{array}\right]- \\
-\sum_{q=0}^{i+n_{2}} T_{i+1, j-q}\left[A_{1} B_{1}\right]\left[\begin{array}{l}
x(0, q) \\
u(0, q)
\end{array}\right]+ \\
+\sum_{p=1}^{+i+n_{1}+1} T_{i-p+1, j+1} \operatorname{Ex}(p, 0)+ \\
+\sum_{q=1}^{j+n_{2}+1} T_{i+1, j-q+1} \operatorname{Ex}(p, 0)
\end{gathered}
$$

It is important to note that (1) does not always have a solution. A necessary and sufficient condition for (1) to have a solution is that the initial conditions (2) satisfy the relation (13) for $(i=$ $0 \& j=0,1,2, \ldots, M)$ and $(j=0 \& i=0,1, \ldots, N)$.

\subsection{The backward solution of general singular} models

Let $\tilde{X}\left(z_{1}, z_{2}\right), \tilde{U}\left(z_{1}, z_{2}\right)$ be respectively the $2-D$ $Z$-transform of the functions $\tilde{x}(i, j)$ and $\tilde{u}(i, j)$. Then by applying the $2-D Z$-transform (Kaczorek, $1985)$ in the dual GSM (12) of (1) we obtain

$$
\begin{gathered}
\tilde{X}\left(z_{1}, z_{2}\right)=\underbrace{\left(\sum_{p=-f_{1}}^{\infty} \sum_{q=-f_{2}}^{\infty} \tilde{T}_{p, q} z_{1}^{-p} z_{2}^{-q}\right)}_{\tilde{G}\left(z_{1}, z_{2}\right)^{-1}} \times \\
\times\left\{\left(B_{0} z_{1} z_{2}+B_{1} z_{2}+B_{2} z_{1}\right) \tilde{U}\left(z_{1}, z_{2}\right)-\right. \\
\quad-B_{0} z_{1} z_{2} \tilde{U}\left(z_{1}, 0\right)-B_{1} z_{2} \tilde{U}\left(z_{1}, 0\right)-
\end{gathered}
$$


$-B_{0} z_{1} z_{2} \tilde{U}\left(0, z_{2}\right)-B_{2} z_{1} \tilde{U}\left(0, z_{2}\right)+B_{0} z_{1} z_{2} \tilde{u}(0,0)$

$+A_{0} z_{1} z_{2} \tilde{x}(0,0)-A_{1} z_{2} \tilde{X}\left(z_{1}, 0\right)-A_{0} z_{1} z_{2} \tilde{X}\left(z_{1}, 0\right)-$

$$
\left.-A_{2} z_{1} \tilde{X}\left(0, z_{2}\right)-A_{0} z_{1} z_{2} \tilde{X}\left(0, z_{2}\right)\right\}
$$

Using the inverse 2-D transformation (Kaczorek, 1985) for (14) and taking into account that $\tilde{T}_{p, q}=$ 0 for $p<-f_{1}$ or $q<-f_{2}$, we obtain

$$
\begin{aligned}
\tilde{x}(i, j) & =\sum_{p=0}^{i+f_{1}+1} \sum_{q=0}^{j+f_{2}+1} \tilde{T}_{i-p+1, j-q+1} B_{0} \tilde{u}(p, q)+ \\
& +\sum_{p=0}^{i+f_{1}} \sum_{q=0}^{j+f_{2}+1} \tilde{T}_{i-p, j-q+1} B_{1} \tilde{u}(p, q)+ \\
& +\sum_{p=0}^{i+f_{1}+1} \sum_{q=0}^{j+f_{2}} \tilde{T}_{i-p+1, j-q} B_{2} \tilde{u}(p, q)+ \\
& -\sum_{p=0}^{i+f_{1}} \tilde{T}_{i-p, j+1}\left[A_{1} B_{1}\right]\left[\begin{array}{l}
\tilde{x}(p, 0) \\
\tilde{u}(p, 0)
\end{array}\right]- \\
& -\sum_{q=0}^{j+f_{2}} \tilde{T}_{i+1, j-q}\left[A_{2} B_{2}\right]\left[\begin{array}{l}
\tilde{x}(0, q) \\
\tilde{u}(0, q)
\end{array}\right]- \\
- & \sum_{p=1}^{i+f_{1}+1} \tilde{T}_{i-p+1, j+1}\left[A_{0} B_{0}\right]\left[\begin{array}{l}
\tilde{x}(p, 0) \\
\tilde{u}(p, 0)
\end{array}\right]- \\
- & \sum_{q=1}^{j+f_{2}+1} \tilde{T}_{i+1, j-q+1}\left[A_{0} B_{0}\right]\left[\begin{array}{l}
\tilde{x}(0, q) \\
\tilde{u}(0, q)
\end{array}\right] \\
& +\tilde{T}_{i+1, j+1}\left[A_{0} B_{0}\right]\left[\begin{array}{l}
\tilde{x}(0,0) \\
\tilde{u}(0,0)
\end{array}\right]
\end{aligned}
$$

Now by using the part (a) of Lemma 2 and the solution of the dual GSM (15) we can easily prove the following Theorem.

Theorem 3. If $\operatorname{det}\left[G\left(z_{1}, z_{2}\right)\right] \neq 0$, then the unique backward solution to (1) with admissible boundary conditions (3) is given by

$$
\begin{gathered}
-\sum_{q=1}^{M-j+\ell_{2}} V_{i-N, q+j-M}\left[\begin{array}{ll}
A_{0} & B_{0}
\end{array}\right]\left[\begin{array}{l}
x(N, M-q) \\
u(N, M-q)
\end{array}\right]+ \\
+V_{i-N, j-M}\left[\begin{array}{ll}
A_{0} & B_{0}
\end{array}\right]\left[\begin{array}{l}
x(N, M) \\
u(N, M)
\end{array}\right]
\end{gathered}
$$

where $V_{i, j}$ is the backward fundamental matrix sequence of $G\left(z_{1}, z_{2}\right)^{-1}$ given in (7).

Proof. Let $\tilde{x}(i, j)$ be the solution of (12) for the non-zero input $\tilde{u}(i, j)$ presented in (15). Then by using (11) and the fact that the sequence $x(i, j)=\tilde{x}(N-i, M-j)$ is a solution of the dual equation (1) for the nonzero input $u(i, j)=$ $\tilde{u}(N-i, M-j)$ from Lemma 2 we get the result.

A necessary and sufficient condition for (1) to have a solution is that the final conditions (3) satisfy (16) for $(i=N \& j=0,1, \ldots, M)$ and $(i=0,1, \ldots ., N \& j=M)$.

3.3 The symmetric solution of general singular models

Consider the Laurent expansion at infinity of $G\left(z_{1}, z_{2}\right)$ given in (6). Then the following relations

$$
\begin{gathered}
T_{p, q} E-T_{p-1, q-1} A_{0}-T_{p, q-1} A_{1}-T_{p-1, q} A_{2}=\delta_{p-1, q-1} I_{n} \\
T_{p, q} E=0_{n, n} \\
\left(\text { for } p<-n_{1} \text { and } / \text { or } q<-n_{2}\right)
\end{gathered}
$$

are following from comparison of coefficient matrices at like powers of $z_{1}$ and $z_{2}$ of the equality

$$
\begin{gathered}
\underbrace{\left(\sum_{p=-n_{1}}^{\infty} \sum_{q=-n_{2}}^{\infty} T_{p, q} z_{1}^{-p} z_{2}^{-q}\right)}_{G\left(z_{1}, z_{2}\right)^{-1}} \times \\
\times \underbrace{\left(z_{1} z_{2} E-A_{0}-A_{1} z_{1}-A_{2} z_{2}\right)}_{G\left(z_{1}, z_{2}\right)}=I_{n}
\end{gathered}
$$

$$
\begin{aligned}
& x(i, j)=\sum_{p=0}^{N-i+\ell_{1}} \sum_{q=0}^{M-j+\ell_{2}} V_{p-i-N, q-j-M} B_{0} u\left(N-p, M \begin{array}{l}
\text { Define now the matrices } \\
\mathcal{A}_{0}=\operatorname{blockdiag}\left(\left(-A_{1}-A_{0}\right), . .,\left(-A_{1}-A_{0}\right)\right)
\end{array}\right. \\
& +\sum^{N-i+\ell_{1}-1} \sum^{M-j+\ell_{2}} \quad \mathcal{A}_{1}=\operatorname{blockdiag}\left(\left(E-A_{2}\right), . .,\left(E-A_{2}\right)\right) \\
& +\sum_{p=0} \sum_{q=0} V_{1+p+i-N, q+j-M} B_{1} u(N-p, M-q)+\mathcal{B}_{0}=\operatorname{blockdiag}\left(\left(B_{1} B_{0}\right), \ldots,\left(B_{1} B_{0}\right)\right) \\
& N-i+\ell_{1} M-j+\ell_{2}-1 \quad \mathcal{B}_{1}=\operatorname{blockdiag}\left(\left(B_{2} 0\right), . .,\left(B_{2} 0\right)\right) \\
& +\sum_{p=0}^{N} \sum_{q=0}^{N-i+\ell_{1}} V_{p+i-N, 1+q+j-M} B_{2} u(N-p, M-q) \text { here } \mathcal{A}_{0} \in R^{n N \times n(N+1)}, \mathcal{A}_{1} \in R^{n N \times n(N+1)}, \mathcal{B}_{0} \in \\
& -\sum_{p=0}^{N-i+\ell_{1}-1} V_{1+p+i-N, j-M}\left[\begin{array}{ll}
A_{1} & B_{1}
\end{array}\right]\left[\begin{array}{l}
x(N-p, M) \\
u(N-p, M)
\end{array}\right]- \\
& -\sum_{q=0}^{M-j+\ell_{2}-1} V_{i-N, 1+q+j-M}\left[A_{2} B_{2}\right]\left[\begin{array}{l}
x(N, M-q) \\
u(N, M-q)
\end{array}\right]- \\
& \begin{array}{c}
y_{i}=\left(\begin{array}{c}
x_{N, i} \\
x_{N-1, i} \\
\vdots \\
x_{1, i} \\
x_{0, i}
\end{array}\right), u_{i}=\left(\begin{array}{c}
u_{N, i} \\
u_{N-1, i} \\
\vdots \\
u_{1, i} \\
u_{0, i}
\end{array}\right) \\
i=0,1, \ldots, M
\end{array}
\end{aligned}
$$$$
-\sum_{p=1}^{N-i+\ell_{1}} V_{p+i-N, j-M}\left[A_{0} B_{0}\right]\left[\begin{array}{l}
x(N-p, M) \\
u(N-p, M)
\end{array}\right]-
$$

where $y_{i} \in R^{(N+1) n}, u_{i} \in R^{(N+1) m}$. Then (1) may be rewritten in the form 


$$
\begin{gathered}
\underbrace{\left(\begin{array}{cccccc}
\mathcal{A}_{1} & \mathcal{A}_{0} & \cdots & 0 & 0 & 0 \\
0 & \mathcal{A}_{1} & \cdots & 0 & 0 & 0 \\
\vdots & \vdots & \ddots & \vdots & \vdots & \vdots \\
0 & 0 & \cdots & \mathcal{A}_{1} & \mathcal{A}_{0} & 0 \\
0 & 0 & \cdots & 0 & \mathcal{A}_{1} & \mathcal{A}_{0}
\end{array}\right)} \underbrace{\left(\begin{array}{c}
y_{M} \\
y_{M-1} \\
\vdots \\
y_{1} \\
y_{0}
\end{array}\right)}_{\tilde{A}_{0, M}}= \\
=\underbrace{\left(\begin{array}{cccccc}
\mathcal{B}_{1} & \mathcal{B}_{0} & \cdots & 0 & 0 & 0 \\
0 & \mathcal{B}_{1} & \cdots & 0 & 0 & 0 \\
\vdots & \vdots & \ddots & \vdots & \vdots & \vdots \\
0 & 0 & \cdots & \mathcal{B}_{1} & \mathcal{B}_{0} & 0 \\
0 & 0 & \cdots & 0 & \mathcal{B}_{1} & \mathcal{B}_{0}
\end{array}\right)}_{\tilde{B}_{N}} \underbrace{\left(\begin{array}{c}
u_{M} \\
u_{M-1} \\
\vdots \\
u_{1} \\
u_{0}
\end{array}\right)}_{v_{0, M}}
\end{gathered}
$$

Let also

$$
\begin{gathered}
\mathcal{H}_{i}=\left(\begin{array}{ccccc}
T_{1, i} & T_{2, i} & \cdots & T_{N-1, i} & T_{N, i} \\
T_{0, i} & T_{1, i} & \cdots & T_{N-2, i} & T_{N-1, i} \\
\vdots & \vdots & \ddots & \vdots & \vdots \\
T_{-N+1, i} & T_{-N+2, i} & \cdots & T_{0, i} & T_{1, i} \\
T_{-N, i} & T_{-N+1, i} & \cdots & T_{-1, i} & T_{0, i}
\end{array}\right) \\
\mathcal{S}_{i}=\left(\begin{array}{ccccc}
F_{1, i} & 0 & \cdots & 0 & Q_{N, i} \\
F_{0, i} & \delta_{i-1} I & \cdots & 0 & Q_{N-1, i} \\
\vdots & \vdots & \ddots & \vdots & \vdots \\
F_{-N+2, i} & 0 & \cdots & \delta_{i-1} I & Q_{2, i} \\
F_{-N+1, i} & 0 & \cdots & 0 & Q_{1, i} \\
F_{k, i}=T_{k, i} E-T_{k, i-1} A_{1}
\end{array}\right. \\
Q_{k, i}=-T_{k, i} A_{2}-T_{k, i-1} A_{0}
\end{gathered}
$$

Then we can check that

$$
\mathcal{H}_{i} \mathcal{A}_{1}+\mathcal{H}_{i-1} \mathcal{A}_{0}=\mathcal{S}_{i}
$$

Premultiplying (18) by the matrix

$$
\tilde{A}_{N}^{L}=\left(\begin{array}{ccccc}
\mathcal{H}_{1} & \mathcal{H}_{2} & \mathcal{H}_{3} & \cdots & \mathcal{H}_{M} \\
\mathcal{H}_{0} & \mathcal{H}_{1} & \mathcal{H}_{2} & \cdots & \mathcal{H}_{M-1} \\
\mathcal{H}_{-1} & \mathcal{H}_{0} & \mathcal{H}_{1} & \cdots & \mathcal{H}_{M-2} \\
\vdots & \vdots & \vdots & \ddots & \vdots \\
\mathcal{H}_{-M+1} & \mathcal{H}_{-M+2} & \mathcal{H}_{-M+3} & \cdots & \mathcal{H}_{0}
\end{array}\right)
$$

we obtain that

$$
\begin{gathered}
\tilde{A}_{N}^{L} \tilde{A}_{N} y_{0, M}=\tilde{A}_{N}^{L} \tilde{B}_{N} v_{0, M} \Leftrightarrow \\
\left(\begin{array}{ccccc}
\mathcal{H}_{1} \mathcal{A}_{1} & \mathcal{S}_{2} & \cdots & \mathcal{S}_{M} & \mathcal{H}_{M} \mathcal{A}_{0} \\
\mathcal{H}_{0} \mathcal{A}_{1} & \mathcal{S}_{1} & \cdots & \mathcal{S}_{M-1} & \mathcal{H}_{M-1} \mathcal{A}_{0} \\
\mathcal{H}_{-1} \mathcal{A}_{1} & \mathcal{S}_{0} & \cdots & \mathcal{S}_{M-2} & \mathcal{H}_{M-2} \mathcal{A}_{0} \\
\vdots & \vdots & \ddots & \vdots & \vdots \\
\mathcal{H}_{-M+1} \mathcal{A}_{1} & \mathcal{S}_{-M+2} & \cdots & \mathcal{S}_{0} & \mathcal{H}_{0} \mathcal{A}_{0}
\end{array}\right) y_{0, M}= \\
=\left(\begin{array}{cccc}
\mathcal{H}_{1} \mathcal{B}_{1} & \mathcal{W}_{1} & \cdots & \mathcal{H}_{M} \mathcal{B}_{0} \\
\mathcal{H}_{0} \mathcal{B}_{1} & \mathcal{W}_{0} & \cdots & \mathcal{H}_{M-1} \mathcal{B}_{0} \\
\mathcal{H}_{-1} \mathcal{B}_{1} & \mathcal{W}_{-1} & \cdots & \mathcal{H}_{M-2} \mathcal{B}_{0} \\
\vdots & \vdots & \ddots & \vdots \\
\mathcal{H}_{-M+1} \mathcal{B}_{1} & \mathcal{W}_{-M+1} & \cdots & \mathcal{H}_{0} \mathcal{B}_{0}
\end{array}\right) v_{0, M}
\end{gathered}
$$

where $\mathcal{W}_{i}=\mathcal{H}_{i} \mathcal{B}_{0}+\mathcal{H}_{i+1} \mathcal{B}_{1}$. From the first and last block equation we get boundary conditions that must be satisfied in order (1) have a solution:

$$
\begin{aligned}
& \mathcal{H}_{1} \mathcal{A}_{1} y_{M}+\mathcal{S}_{2} y_{M-1}+\cdots+\mathcal{S}_{M} y_{1}+\mathcal{H}_{M} \mathcal{A}_{0} y_{0}= \\
& =\left(\mathcal{H}_{1} \mathcal{B}_{1}\right) u_{M}+\left(\mathcal{H}_{1} \mathcal{B}_{0}+\mathcal{H}_{2} \mathcal{B}_{1}\right) u_{M-1}+\cdots+ \\
& \quad+\left(\mathcal{H}_{M-1} \mathcal{B}_{0}+\mathcal{H}_{M} \mathcal{B}_{1}\right) u_{1}+\left(\mathcal{H}_{M} \mathcal{B}_{0}\right) u_{0}
\end{aligned}
$$

and

$\mathcal{H}_{-M+1} \mathcal{A}_{1} y_{M}+\mathcal{S}_{-M+2} y_{M-1}+\cdots+\mathcal{S}_{0} y_{1}+\mathcal{H}_{0} \mathcal{A}_{0} y_{0}=$ $=\left(\mathcal{H}_{-M+1} \mathcal{B}_{1}\right) u_{M}+\left(\mathcal{H}_{-M+1} \mathcal{B}_{0}+\mathcal{H}_{-M+2} \mathcal{B}_{1}\right) u_{M-1}+$ $+\cdots+\left(\mathcal{H}_{0} \mathcal{B}_{0}+\mathcal{H}_{1} \mathcal{B}_{1}\right) u_{1}+\left(\mathcal{H}_{0} \mathcal{B}_{0}\right) u_{0}$

Note that the matrices $S_{i}, i=0,2,3, \ldots, M$ in (20) and (21) have all their block columns, except of the first and the last one, filled with zero entries and therefore the above equations gives rise only to boundary conditions of the form (4). Now consider the rest equations that are coming from (19)

$$
\begin{gathered}
\quad\left(\mathcal{H}_{-q} \mathcal{A}_{1}\right) y_{M}+\mathcal{S}_{-q+1} y_{M-1}+\cdots+ \\
\quad+\mathcal{S}_{-q+M-1} y_{1}+\left(\mathcal{H}_{-q+M-1} \mathcal{A}_{0}\right) y_{0}= \\
=\left(\mathcal{H}_{-q} \mathcal{B}_{1}\right) u_{M}+\left(\mathcal{H}_{-q} \mathcal{B}_{0}+\mathcal{H}_{-q+1} \mathcal{B}_{1}\right) u_{M-1}+ \\
+. .+\left(\mathcal{H}_{-q+M-1} \mathcal{B}_{0}+\mathcal{H}_{-q+M-1} \mathcal{B}_{1}\right) u_{1}+\left(\mathcal{H}_{-q+M-1} \mathcal{B}_{0}\right) u_{0}
\end{gathered}
$$

where $q=0,1, \ldots, M-2$, by taking the $i$-th row of the above equations i.e. for $q=0,1, \ldots, M-2$ and $i=0,1, \ldots, N-2$ and by substituting $N-1+i$ with $p$, and $M-1-q$ with $q$, we can easily get the following Theorem.

Theorem 4. If $\operatorname{det}\left[G\left(z_{1}, z_{2}\right)\right] \neq 0$, and $\operatorname{deg}_{z}|G(z, z)|=$ $\operatorname{deg}_{z_{1}}\left|G\left(z_{1}, z_{2}\right)\right|+\operatorname{deg}_{z_{2}}\left|G\left(z_{1}, z_{2}\right)\right|$ is satisfied, then the unique symmetric solution to (1) with admissible boundary conditions (2) is given by

$$
\begin{gathered}
x_{p, q}=-T_{N-1-p, 1+q-M} E x_{N, M}+ \\
+T_{2(N-1)-p, 1+q-M} A_{2} x_{0, M}+ \\
+\sum_{k=0}^{N-2}\left\{T_{N-1-p+k, 1+q-M} A_{2}-\right. \\
\left.-T_{N-p+k, 1+q-M} E\right\} x_{N-1-k, M}+ \\
+\sum_{j=q+2-M}^{q+1}\left\{\left\{-T_{N-1-p, j} E+\right.\right.
\end{gathered}
$$

$\left.+T_{N-1-p, j-1} A_{1}\right\} x_{N, q+2-j}+\left\{-T_{2(N-1)-p, j} A_{2}-\right.$ $\left.\left.-T_{2(N-1)-p, j-1} A_{0} x_{0, q+2-j}\right\}\right\}+T_{N-1-p, q} A_{1} x_{N, 0}+$ $+\sum_{k=0}^{N-2}\left\{T_{N-1-p+k, q} A_{0}+T_{N-p+k, q} A_{1}\right\} x_{N-1-k, 0}+$ $+T_{2(N-1)-p, q} A_{0} x_{0,0}+$ $+\sum_{k=0}^{N-1} T_{N-1-p+k, q+1-M} B_{2} u_{N-k, M}+$ $+\sum_{j=q+1-M}^{q-1} \sum_{k=0}^{N-2}\left\{T_{N-1-p+k, j} B_{0}+T_{N-p+k, j} B_{1}+\right.$ $\left.+T_{N-p+k, j+1} B_{2}\right\} u_{N-k-1, M-1-j}+$

$+\sum_{j=q+1-M}^{q-1}\left\{\left(T_{N-1-p, j} B_{1}+T_{N-1-p, j+1} B_{2}\right) u_{N, M-1-j}+\right.$ $\left.+\left(T_{2(N-1)-p, j} B_{0}+T_{2(N-1)-p, j+1} B_{2}\right) u_{0, M-1-j}\right\}+$ 


$$
\begin{aligned}
& +\left(T_{N-1-p, q} B_{1}\right) u_{N, 0}+\left(T_{2(N-1)-p, q} B_{0}\right) u_{0,0}+ \\
& +\sum_{k=0}^{N-2}\left\{T_{N-1+p+k, q} B_{0}+T_{N-p+k, q} B_{1}\right\} u_{N-1-k, 0}
\end{aligned}
$$

Using now the first and last block row equations of (22) we get the following extra boundary conditions for $(i=-1, N-1 \& q=0,1, \ldots, M-2)$, or $(q=-1, M-1 \& i=-1,0, \ldots, N-2, N-1)$

$$
\begin{aligned}
& T_{-i,-q} E x_{N, M}-T_{-i+N-1,-q} A_{2} x_{0, M}+ \\
& +\sum_{k=0}^{N-2}\left(-T_{-i+k,-q} A_{2}+T_{-i+k+1,-q} E\right) x_{N-1-k, M}+ \\
& +\sum_{j=1-q}^{M-q}\left\{\left(T_{-i, j} E-T_{-i, j-1} A_{1}\right) x_{N, M-q+1-j}+\right. \\
& \left.+\left(-T_{-i+N-1, j} A_{2}-T_{-i+N-1, j-1} A_{0}\right) x_{0, M-q+1-j}\right\}- \\
& -T_{-i, M-1-q} A_{1} x_{N, 0}- \\
& -\sum_{k=0}^{N-2}\left(\begin{array}{c}
T_{-i+k, M-1-q} A_{0}+ \\
+T_{-i+k+1, M-1-q} A_{1}
\end{array}\right) x_{N-1-k, 0}- \\
& -T_{-i+N-1, M-1-q} A_{0} x_{0,0}=\sum_{k=0}^{N-1} T_{-i+k,-q} B_{2} u_{N-k, M}+ \\
& +\sum_{j=-q}^{M-2-q} \sum_{k=0}^{N-2}\left\{T_{-i+k, j} B_{0}+T_{-i+k+1, j} B_{1}+\right. \\
& \left.+T_{-i+k+1, j+1} B_{2}\right\} u_{N-k-1, M-1-j}+ \\
& +\sum_{j=-q}^{M-2-q}\left\{\left(T_{-i, j} B_{1}+T_{-i, j+1} B_{2}\right) u_{N, M-1-j}+\right. \\
& \left.+\left(T_{-i+N-1, j} B_{0}+T_{-i+N-1, j+1} B_{2}\right) u_{0, M-1-j}\right\} \\
& +\left(T_{-i, M-1-q} B_{1}\right) u_{N, 0}+\left(T_{-i+N-1, M-1-q} B_{0}\right) u_{0,0}+ \\
& +\sum_{k=0}^{N-2}\left(\begin{array}{c}
T_{-i+k, M-1-q} B_{0}+ \\
+T_{-i+k+1, M-1-q} B_{1}
\end{array}\right) u_{N-1-k, 0}
\end{aligned}
$$

Therefore, a necessary and sufficient condition so that (1) has a solution is that the initial conditions, final conditions and input sequences satisfy the relations (4), (20), (21) and (23).

\section{CONCLUSIONS}

In the case of general discrete time singular models, exact solutions where proposed in two different forms : a) backward solutions, and b) symmetric solutions. All the closed formula solutions were represented in terms of the forward and backward fundamental matrix of the GSM. It is easily seen that the proposed solutions : a) are extensions of the ones proposed in (Lewis and Mertzios, 1990) for 1-D discrete time singular systems, and b) accomplish the work that have been done by (Kaczorek, 1990) and (Lewis and Mertzios, 1991) for the forward solution of the GSM and the implicit Roesser model respectively.
Certain controllability and observability criteria based on the proposed solutions are being studied and will be discussed in a future work.

\section{REFERENCES}

Fornasini, E. and G. Marchesini (1970). Doubly indexed dynamical systems : State space models and structural properties. Math. Systems Theory 12, 59-72.

Kaczorek, T. (1985). Two-Dimensional Linear Systems. Springer Verlag, Berlin.

Kaczorek, T. (1987). Singular roesser model and reduction to its canonical form. Bulletin Polish Academy of Sciences, Technical Sciences 35, 645-652.

Kaczorek, T. (1988). The singular general model of 2-d systems and its solution. IEEE Transaction on Automatic Control 33, 1060-1061.

Kaczorek, T. (1989). Equivalence of singular 2d linear models. Bull. Polish Academy of Sciences.

Kaczorek, T. (1990). General response formula and minimum energy control for the general singular model of 2-d systems. IEEE Trans. on Automatic Control 35(4), 433-436.

Karamancioglu, A. (1991). Two-Dimensional Implicit Linear Systems. PhD thesis. Dept. of Electrical Engineering, University of Texas at Arlington.

Karampetakis, N.P., B.G. Mertzios and A.I.G. Vardulakis (1994). Computation of the transfer function matrix and its laurent expansion of generalized two-dimensional systems. International Journal of Control 60(4), 521541.

Kurek, J. (1985). The general state-space model for a two-dimensional linear digital system. IEEE Trans. on Automatic Control 30, 600602 .

Lewis, F. L. and B.G. Mertzios (1991). On the analysis of two-dimensional discrete singular systems. Circuit Systems and Signal Proc.

Lewis, F.L. (1987). Recent work in singular systems. In: Proc. Int. Symp. Singular Systems. Atlanta, GA. pp. 20-24.

Lewis, F.L. (1992). A review of 2-d implicit systems. Automatica 28(2), 345-354.

Lewis, F.L. and B.G. Mertzios (1990). On the analysis of discrete linear time-invariant singular systems. IEEE Trans. on Automatic Control 35(4), 506-511.

Mertzios, B.G. and F.L. Lewis (1988). An algorithm for the computation of the transfer function matrix of generalized 2-d systems. Circuit Systems and Signal Process 7, 459466.

Roesser, R.P. (1975). A discrete state-space model for linear image processing. IEEE Trans. on Automatic Control 25, 1-10. 